\title{
Synchrotron and Inverse Compton radiation from relativistic plasmas. Effects of coherence and turbulence
}

\section{Guy Pelletier*t}

Laboratoire d'Astrophysique de Grenoble, France

E-mail: Guy.Pelletierdobs.ujf-grenoble.fr

Compact object environments such as those of Black Holes and Pulsars display relativistic outflows that collide with the ambient medium and generate very high energy particles revealed by their synchrotron and inverse Compton radiations. These continuum emissions are very similar especially at the highest energies where they allow to determine the electron distribution. Whereas the very high energy part of the spectrum is very likely produced by an inverse Compton process, the low energy part is abusively considered as resulting from a synchrotron emission. However the physics of relativistic shocks do not infer that conclusion and it could be due to some kind of coherent or incoherent inverse Compton emission by scattering off collective plasma waves. These two radiation mechanisms could be discriminated through coherence measurements, because the standard synchrotron emission is not responsible for radiation field correlation, whereas the inverse Compton field display correlations due to its nonlinearity. The possibility to make such measurements in a near future with the Pulsar Wind Nebula of Crab Nebula as a favorite target would opened an interesting window in the astrophysics of compact object environments.

Quantum of Quasars workshop - QQ09,

December 2-4, 2009

Grenoble, France

* Speaker.

$\dagger$ A footnote may follow. 


\section{Preamble}

Relativistic ejections of plasmas take place in the environment Black Holes and Pulsars and their collision with the ambient medium gives rise to relativistic shocks. These shocks produce very high energy particles that radiate through synchrotron and Inverse Compton emissions. Synchrotron emission in a large scale static field has a well known signature that allows to deduce some parameters of the high energy source. Often these sources radiate another continuous spectrum with a similar shape at much higher energy, which is interpreted as an Inverse Compton emission due to the relativistic electrons scattering off low energy synchrotron-photons. However the interpretation of the low energy spectrum as synchrotron emission is not unambiguous. Indeed IC-radiation through an intense coherent electromagnetic wave produce a spectrum similar to a synchrotron one. This is also the same for IC-emission off plasma micro-turbulence, except that the low frequency part of the spectrum increases more steepily than an synchrotron one. It turns out that in the relativistic shock of those flows, the mean field is not strong enough to account for the "synchrotron" emission and either it is amplified in the form of large scale turbulence, or another type of short scale electromagnetic turbulence is generated without any relation with the mean field, or even an intense coherent electromagnetic wave is generated. The generation of electromagnetic disturbances gives rise to a particular kind of IC-radiation, which looks like a synchrotron emission except sometimes at low frequencies. As opposed to synchrotron emission, IC-emission is nonlinear and could be discriminated by the measurement of coherence functions $g^{(1)}$ and $g^{(2)}$.

\section{Classical laws of radiation by a moving particle}

A moving charged particle radiates an electromagnetic field that can be decomposed into a localized part proportional to the velocity of the particle, with an electric field decreasing with distance from the particle like $1 / r^{2}$ and a delocalized part proportional to the acceleration of the particle, the radiation field, that decreases like $1 / r$. The radiation field has a magnetic counterpart $\vec{B}=\vec{n} \times\left.\vec{E}\right|_{t^{\prime}}$, where $t^{\prime}=t-r\left(t^{\prime}\right) / c$; and the associated Poynting vector $\vec{S}=\frac{c}{4 \pi} \vec{E} \times \vec{B}$ decreases with distance like $1 / r^{2}$ and thus leads to a constant power radiated to infinity. For a non-relativistic particle, the radiation field is (Rybicki \& Lightman 1979, Jackson 1999)

$$
\vec{E}=\frac{e}{c^{2} r\left(t^{\prime}\right)} \vec{n} \times(\vec{n} \times \dot{\vec{v}})
$$

where $\vec{n}$ is the unit vector generating the direction of the line of sight. The field is polarized in the direction of the projection of the acceleration transverse to the line of sight (since $\vec{n} \times(\vec{n} \times \dot{\vec{v}})=$ $-\dot{\vec{v}}_{\perp}$ ) and the power radiated to infinity per element of solid angle $d \Omega$ around the direction of the light of sight is given by the celebrated Larmor formula:

$$
\frac{d P}{d \Omega}=\frac{e^{2}}{4 \pi c^{3}}|\vec{n} \times(\vec{n} \times \dot{\vec{v}})|^{2} .
$$

The total power radiated in all directions is

$$
P=\frac{2}{3} \frac{e^{2}}{c^{3}}|\dot{\vec{v}}|^{2}
$$


The extension of these formulae for a particle in relativistic motions is obtained as follows. One can define

$$
r\left(t^{\prime}\right) \vec{E} \equiv \frac{e}{c} \vec{A}
$$

where

$$
\vec{A}(t)=\frac{\vec{n}}{g^{3}} \times\left.[(\vec{n}-\vec{\beta}) \times \dot{\vec{\beta}}]\right|_{t^{\prime}}
$$

with $t^{\prime}=t-r\left(t^{\prime}\right) / c$ and $g \equiv 1-\vec{\beta} \cdot \vec{n}$. In the non-relativistic case $\vec{A}$ is the projection of the acceleration in the direction transverse to the line of sight: $\vec{A}=\vec{n} \times(\vec{n} \times \dot{\vec{\beta}})$. And the instantaneous power emitted per element of solid angle $d \Omega$ :

$$
\frac{d P}{d \Omega}=\frac{e^{2}}{4 \pi c}|\vec{A}(t)|^{2}
$$

The factor $g$ gives the relation between the emission and the reception times measured by the observer: $d t_{r}=g d t_{e}$ (or simply $d t=g d t^{\prime}$ ). The important particularity of the relativistic radiation is that the parameter $g$ can becomes small when the particle speed points towards the observer, so that $g \simeq \frac{1}{2}\left(\frac{1}{\gamma^{2}}+\theta^{2}\right)$. This occurs during a laps time $\Delta t_{e}$ during which the speed angle $\theta$ with respect to the line of sight varies of an amount $1 / \gamma$. During the time laps of strong emission $g \sim 1 / \gamma^{2}$ and the pulse is received by the observer during a much shorter time $\Delta t_{r}$ such that $\Delta t_{r} \sim \Delta t_{e} / \gamma^{2}$. Moreover during that strong pulse emission, the radiation field can be simplified to:

$$
\vec{A}(t) \simeq-\left.\frac{\dot{\vec{\beta}}_{\perp}}{g^{2}}\right|_{t^{\prime}},
$$

the next contributions being of order $1 / \gamma^{2}$ smaller.

\section{Properties of radiations by a relativistic particle}

- For equivalent intensities, the radiative contribution of the force component parallel to the velocity is smaller than the contribution of the perpendicular component by a factor $1 / \gamma^{2}$. (it can be shown with the general formula of the power radiated by a particle in relativistic motions, given in the next section, that i) for a linear trajectory $P=\frac{2}{3} \frac{e^{2}}{m^{2} c^{3}}|\dot{\vec{p}}|^{2}$ whereas ii) for a circular trajectory $P=\frac{2}{3} \frac{e^{2}}{m^{2} c^{3}} \gamma^{2}|\dot{\vec{p}}|^{2}$.)

- Radiated photons are concentrated in a cone of half angle $1 / \gamma$ in the direction of the particle velocity.

- The particle radiation emission occurs during very short intervals when the particle velocity points towards the observer within a cone of half angle $1 / \gamma$.

- During the perception of the particle emission, its trajectory can often (except in some special situations, that will be presented further on) be assimilated to a circular path of curvature radius $\rho$ such that

$$
-\frac{\vec{e}_{t}}{\rho}=\frac{\dot{\vec{v}}_{\perp}}{v^{2}} \simeq \frac{\dot{\vec{\beta}}_{\perp}}{c} .
$$


- The duration of the emission within the cone of half angle $1 / \gamma$ is

$$
\Delta t_{e} \sim \rho / \gamma \nu
$$

The corresponding trajectory (almost straight) length is thus $d \sim \rho / \gamma$.

- The length of the light pulse perceived by the observer is thus

$$
\ell_{r}=(c-v) \Delta t_{e} \simeq \frac{1-\beta}{\beta \gamma} \rho \simeq \rho / 2 \gamma^{3} .
$$

- The duration of the reception of the pulse by the observer is thus

$$
\Delta t_{r} \sim \frac{\rho}{2 \gamma^{3} c} \sim \frac{\Delta t_{e}}{2 \gamma^{2}} .
$$

- The characteristic frequency of the emission is thus

$$
\omega_{r} \sim 2 \gamma^{3} \frac{c}{\rho}
$$

An important example of this type of radiation is synchrotron emission in an uniform magnetic field $B_{0}$ with $\rho=r_{L} / \sin \alpha$ (where the Larmor radius is $r_{L} \equiv \gamma m c^{2} / e B_{0}$, and $\alpha$ the pitch angle of the particle with respect to the direction of the magnetic field). The characteristic frequency of the synchrotron emission is thus

$$
\omega_{s y n}=2 \gamma^{2} \frac{e B}{m c} .
$$

The other important example is the strong Inverse Compton radiation (by "strong" I mean that the particle has not time to ascillate when crosing the emission cone; the criterium will be given in section 5). Its characteristics are the same by substituting $E_{0}$ to $B_{0}$. The emitted power is the same

$$
P \sim \sigma_{T} c \frac{B_{0}^{2} \text { or } E_{0}^{2}}{4 \pi} \gamma^{2}
$$

where the Thomson cross section $\sigma_{T}=\frac{8 \pi}{3} r_{e}^{2}$ with the electron classical radius $r_{e} \equiv \frac{e^{2}}{m_{e} c^{2}}$.

For both types of radiation the emissivity decreases exponentially for $\omega \gg \omega_{r}$ (more precisely as $\left(\frac{\omega}{\omega_{r}}\right)^{1 / 2} \exp \left[-\frac{\omega}{\omega_{r}}\right]$. The emission at $\omega \ll \omega_{r}$ is an interesting issue.

\section{Calculation of the emission by a relativistic particle}

The Fourier transform of the radiation field leads to

$$
\hat{\vec{A}}(\omega)=\int_{\mathbb{R}} \frac{\vec{n}}{g^{2}} \times[(\vec{n}-\vec{\beta}) \times \dot{\vec{\beta}}] \exp \left[i \omega\left(t^{\prime}-\vec{n} \cdot \vec{x}\left(t^{\prime}\right) / c\right)\right] d t^{\prime} .
$$

The time dependence in the exponential comes from $t=t^{\prime}+r\left(t^{\prime}\right) / c$ with $r\left(t^{\prime}\right) \simeq r-\vec{n} \cdot \vec{x}\left(t^{\prime}\right)$. During the short time emission,

$$
\hat{\vec{A}}(\omega) \simeq-\int_{\mathbb{R}} \dot{\vec{\beta}}_{\perp} \exp \left[i \omega\left(t^{\prime}-\vec{n} \cdot \vec{x}\left(t^{\prime}\right) / c\right)\right] \frac{d t^{\prime}}{g} .
$$


One can approximate $\dot{\vec{\beta}}_{\perp} \simeq-\frac{c \vec{e}_{t}}{\rho}$ and the phase can be expanded such that

$$
t^{\prime}-\vec{n} \cdot \vec{x}\left(t^{\prime}\right) / c=\frac{1}{2}\left(\frac{1}{\gamma^{2}}+\theta^{2}\right) t^{\prime}+\frac{c^{2}}{6 \rho^{2}} t^{\prime 3}+\mathscr{O}\left(\Delta t_{e} / \gamma^{4}\right) .
$$

The result can be expressed in term of Airy integrals (see Jackson 1999).

The two retained contributions in the phase are comparable for $t^{\prime} \sim \Delta t_{e} \sim \rho / c \gamma$. As long as the curvature can be considered as constant, which is exact for normal synchrotron emission, the contribution in $t^{13}$ leads to low frequency dependance in $\omega^{1 / 3}$ for long integration time compared to $\Delta t_{e}$.

In the case of synchrotron emission by a single electron, the precise calculation of the emissivity leads to a power spectrum of the form:

$$
\frac{d P}{d \omega}=c_{0} \frac{P}{\omega_{*}} F\left(\frac{\omega}{\omega_{*}}\right),
$$

with

$$
\begin{aligned}
F(x) & =x \int_{x}^{\infty} K_{5 / 3}(y) d y \\
\omega_{*} & =\frac{3}{2} \gamma^{2} \omega_{c} \sin \alpha \\
c_{0} & =\frac{9 \sqrt{3}}{4}
\end{aligned}
$$

The function $F$ reaches a maximum for $x_{0}=0.29$ with $F\left(x_{0}\right)=0.92$, and it is quite remarkable that $x_{0} \omega_{*}$ is very close to the phenomenological estimate $\omega_{r} . \mathrm{P}$ is the total power emitted by the particle after angular integration:

$$
P=\frac{4}{3} \sigma_{T} \frac{B^{2}}{8 \pi} \gamma^{2}
$$

\section{Inverse Compton emission}

Interaction of relativistic electrons with soft photons generates high energy photons through single or multiple scattering. In general in non-thermal sources, unless in Klein-Nishina regime, Inverse Compton emission refers to the single Thomson scattering that occurs in the electron rest frame. This is a linear process, but nonlinear induced scatterings can occur when the primary waves have high intensity; these are the double, triple, multiple scattering involving higher and higher degree of nonlinearity (like in the LPM effect). The parameter of nonlinearity is

$$
a \equiv \frac{e E_{0}}{m_{e} c \omega_{0}},
$$

where $E_{0}$ is the quadratic average amplitude of the primary field and $\omega_{0}$ its characteristic pulsation (let note that the mass is not multiplied by the Lorentz factor $\gamma$ of the particle this usual definition; however it will be relevant to introduce it in section 8). The parameter $a$ measures the intensity of the vibration of the electrons in the wave. For $a>1$, the electron oscillations in the wave are relativistic and the Inverse Compton effect is highly nonlinear and no oscillation occurs within the emission cone crossing since $\omega_{0} \Delta t_{e}<1$. 
In the highly nonlinear regime, Inverse Compton emission is similar to synchrotron emission. In strong regime, the characteristic frequency has to be estimated with the rms intensity of the primary field:

$$
\omega_{r} \simeq 2 \gamma^{2} \frac{e<E^{2}+B^{2}>^{1 / 2}}{m_{e} c} .
$$

The behavior of the emissivity is the same as the regular synchrotron emission for frequency $\omega \geq \omega_{r}$ and for $\omega \ll \omega_{r}$ it grows in $\omega^{1 / 3}$ like synchrotron one. The calculation of the nonlinear emission in a monochromatic wave has been performed for arbitrary value of the parameter $a$ at the beginning of the seventies (Rees 1971, Gun \& Ostriker 1971, Blandford \& Rees 1972, Arons 1972, etc.). This is a tedious calculation and the result quite complicated, even if we try to expand it in series of powers of $a$.

For small value of $a$, electrons have time to oscillate when crossing the emission cone; the leading order contribution to the radiation field produced by the small amplitude vibrations of the electrons in the waves leads to the usual Inverse Compton radiation, the relativistic version of the Thomson effect (whereas the Thomson effect is a pure scattering with energy conservation, the relativistic version of the Inverse Compton effect, leads to an energy variation as measured in the observer frame). The results is the same as this obtained from a quantum derivation in terms of collisions with photons, provided that the condition for Thomson scattering is fulfilled in the electron rest frame, namely $\left.\hbar \omega_{s}\right|_{\text {rest frame }} \ll m_{e} c^{2}$. In weak regime, the power emitted per solid angle is easily calculated from eq.(4.2) with

$$
\dot{\vec{\beta}} \simeq \frac{e}{m_{e} c \gamma}\left[\left(1-\vec{\beta} \cdot \vec{n}_{0}\right) \vec{E}_{0}+\left(\vec{\beta} \cdot \vec{E}_{0}\right) \vec{n}_{0}\right] \cos \left(\left(\omega_{0}-\vec{k}_{0} \cdot \vec{v}\right) t^{\prime}+\phi_{0}\right),
$$

for a linearly polarized mode. This result can be extended for an isotropic spectrum such that $\int S\left(k_{0}\right) d k_{0}=\left|\vec{E}_{0}\right|^{2}$ :

$$
\frac{d^{2} P}{d \Omega d \omega}=\frac{\sigma_{T}}{16 \pi} c \gamma^{2} \int d k_{0} S\left(k_{0}\right) \int \frac{d \Omega_{0}}{4 \pi} g_{0}^{2} \delta\left(\omega-\omega_{0} g_{0} \gamma^{2}\right)
$$

where $g_{0} \equiv 1-\eta_{0} \vec{n}_{0} \cdot \vec{\beta}$.

In the vacuum, the refractive index $\eta_{0}=1$ and the angular average of $g_{0}^{2}$ is $<g_{0}^{2}>=1+\beta^{2} / 3$. The integration over the emitted frequencies leads to

$$
P \sim \sigma_{T} c \gamma^{2} \frac{<E^{2}>}{4 \pi} .
$$

the characteristic frequency of the emission is $\omega_{*}=\frac{1}{2} \gamma^{2} \bar{\omega}_{0}$, where $\bar{\omega}_{0}$ is the frequency at the maximum of the spectrum of low frequency waves. The emission per frequency band, integrated over a population of relativistic electrons, is of the form

$$
S_{\text {rad }}(\omega)=\frac{\sigma_{T} c}{\bar{\omega}_{0}} \frac{<E^{2}>}{4 \pi} \int f\left(\frac{\omega}{\bar{\omega}_{0} \gamma^{2}}\right) \rho(\gamma) d \gamma .
$$

Because of the integration of the delta-function over the angles, the function $f(x)$ has a compact support. Similar to synchrotron emission, for a power law energy spectrum of electrons $\rho(\gamma) \propto \gamma^{-s}$, the high energy (i.e. $\omega \gg \omega_{*}$ ) emitted spectrum is $\propto \omega^{-\frac{s-1}{2}}$. Contrary to synchrotron emission, the low energy (i.e. $\omega \ll \omega_{*}$ ) spectrum is not $\propto \omega^{1 / 3}$; it grows much steeper. 
In a plasma with electromagnetic waves of large refractive index (like Alfvén and whistler waves for instance), $g_{0} \sim \eta_{0}$, the spectrum of the electric field is replaced by the spectrum of the magnetic field of the waves, through the multiplication by $\eta_{0}^{2}$. The characteristic frequency of the radiation is

$$
\omega_{*}=\frac{1}{2} \gamma^{2} \bar{\omega}_{0} \eta_{0}=\frac{1}{2} \gamma^{2} c / \ell_{c}
$$

The coherence length of the magnetic fluctuations $\ell_{c}$ can be as short as the inertial length of the electrons $\delta_{e}=c / \omega_{p e}$. The emitted spectrum looks like a synchrotron spectrum, except at low frequencies where the growth is steeper than $\omega^{1 / 3}$. The integrated power is the same as the synchrotron power in which the energy density of the ordered magnetic field is replaced by that of the fluctuating field.

In the case of electrostatic waves, like Langmuir waves, whose phase velocity can be either smaller or larger than the velocity of light, the properties of the emission is very similar to the IC-emission, with a characteristic frequency $\omega_{*} \sim \gamma^{2} \omega_{p e}$ when most of the Langmuir waves have sub-luminal phase velocity $\left(\eta_{0} \leq 1\right)$ and $\sim \gamma^{2} c / \ell_{c}$ (when $\ell_{c}<\delta_{e}$ ) otherwise (in agreement with Melrose 1980).

When one has to discriminate an emission spectrum, that could result from a kind of inverse Compton process or a scattering off irregular magnetic field, from a standard synchrotron spectrum, only the low frequency part of the spectrum can be significantly changed. This is because longer and longer integration can allow the accumulation of several wiggles of the trajectory in the emission cone and therefore the previous approximation with a single curvature is ruled out. When this is produced by an irregular quasi static magnetic field, it has been called "jitter radiation" (Medvedev 00) and the calculation is different than the one above presented for the weakly nonlinear regime.

\section{Jitter radiation}

Jitter radiation (Medvedev 2000) occurs in synchrotron emission when the magnetic field is strongly varying at scales shorter than Larmor scale. The pitch angle variation during a travel over a field correlation length $\ell_{c}$ is $\Delta \alpha=\frac{\left|\Delta p_{\perp}\right|}{p} \sim \frac{e B_{\perp} \ell_{c}}{\gamma m_{e} c^{2}}$. The ratio of the pitch angle deflection over the radiation cone angle is $\gamma \Delta \alpha \sim \frac{e \bar{B} \ell_{c}}{m_{e} c^{2}}$. This ratio is similar to the nonlinear parameter $a$. This means that for weak nonlinearity such that the coherence length $\ell_{c}$ be smaller than the kind of Larmor radius $r_{0} \equiv m_{e} c^{2} / e \bar{B}$, the electron trajectory can undergo many wiggles within the radiation cone. This is the special regime of jitter radiation. This occurs also with inverse Compton radiation when the nonlinearity is weak. Actually Fermi particle acceleration develops at relativistic shocks when the parameter $a$ is small.

The pulse duration received by the observer is $\Delta t_{j} \sim \frac{\ell_{c}}{\gamma^{2} c}$ and the characteristic frequency of the emission is $\omega_{j} \sim \gamma^{2} c / \ell_{c}$. For frequencies much larger than the characteristic frequencies, the power emission is similar to the normal synchrotron or IC emission. For lower frequency the behavior is different, it increases proportionally to $\omega$. A precise calculation of this effect is difficult and could be done with the path integral technique. 
Severe limitation to this process of jitter radiation has recently been calculated (Kirk \& Reville 2010); the maximum photon energy that can be emitted by this process in about $40 \mathrm{eV}$ in the comoving frame of the relativistic flow.

\section{Coherence effects}

For a power law energy distribution of the relativistic electrons, the spectrum is distributed over twice more decades of energy than the electron energy distribution. The coherence time is determined by the lowest characteristic frequency $\sim \gamma_{\min }^{2} \omega_{* *}$.

The radiation process can display nonlinear effect; this is clear when IC process is due to an intense field that produces multiple scattering. The single scattering generates a random phase radiation field that leads to a Gaussian stochastic process. Multiple scattering for small $a$ can be described by a radiation field expanded in powers of the lowest order Gaussian field organized with the expansion parameter $a$ :

$$
\vec{A}=A_{0} \vec{Y} \text { with } \vec{Y}=\vec{X}+a \Phi_{2}(\vec{X}, \vec{X})+\ldots
$$

The lowest order approximation satisfies

$$
g^{(2)}(X)=1+\left|g^{(1)}(X)\right|^{2},
$$

where the correlations functions of first and second order of the random process $X(t)$ are defined by

$$
\begin{aligned}
g^{(1)}(X, \tau) & =\frac{<X^{*}(t) X(t+\tau)>}{<|X|^{2}>} \\
g^{(2)}(X, \tau) & =\frac{<X^{*}(t) X^{*}(t+\tau) X(t+\tau) X(t)>}{\left(<|X|^{2}>\right)^{2}} .
\end{aligned}
$$

The previous relation between $g^{(1)}$ and $g^{(2)}$ characterizes a process that does not reveal any coherence effect. The process $Y$ has a coherence effect due to the nonlinearity:

$$
g^{(2)}(Y)=1+\left|g^{(1)}(Y)\right|^{2}+a^{2} \mathscr{C}_{3}\left(g^{(1)}, g^{(1)}, g^{(1)}\right)+\ldots
$$

The measurement of $g^{(2)}$ would allow to discriminate an IC-emission from a synchrotron emission and to know the amplitude parameter $a$. Knowing independently $B_{0}$ or $E_{0}$, we could deduce the correlation length.

What are the correlations functions of the radiation field for larger $a$ ? This is not known at all.

\section{Radiation from astrophysical shocks}

In SNR shocks and in relativistic shocks, like those of GRBs, intense turbulence is generated upstream and transmitted downstream. This level of electromagnetic turbulence can far exceed the level of mean field energy density and thus the radiation is due to motions of relativistic electrons in turbulent fields. In the case of SNR shocks, turbulence is mostly at MHD scale and of Alfvénic type, whereas in relativistic shock micro-turbulence is intensively excited. 
The radiation spectrum looks almost similar to the standard synchrotron emission spectrum, except at low frequencies, especially when the electron oscillation regime is weak. The strength of electron collective oscillation in a wave is characterized by a similar nonlinearity parameter $a$ defined with an average Lorentz factor $\bar{\gamma}$ (associated to the relativistic enthalpy of the electron fluid) by

$$
a \equiv \frac{e E_{0}}{m_{e} \bar{\gamma} c \omega_{0}}
$$

When the electric field is associated with an intense magnetic component of an electromagnetic wave, it is convenient to multiply numerator and denominator by the large refractive index and this becomes:

$$
a=\frac{e \bar{B} \ell_{c}}{m_{e} \gamma c^{2}}=\frac{\ell_{c}}{\bar{r}_{L e}}
$$

where $\bar{r}_{L e}$ is the Larmor radius of the electron fluid with "thermal" Lorentz factor $\bar{\gamma}$.

\subsection{Radiation from non-relativistic shocks}

Intense Magneto-Hydro-Dynamic turbulence is generated upstream SNR shocks, such that the turbulent field is much larger than the mean field. The coherence length is larger than $\frac{c}{\omega_{p i}}$. The nonlinearity parameter is likely strong, especially for the low energy electrons responsible for the low frequency emission. The emission spectrum is thus similar to a synchrotron spectrum even at low frequency. The characteristic emission frequency is therefore:

$$
\omega_{*}=\frac{1}{2} \gamma^{2} \frac{e \bar{B}}{m_{e} c}
$$

The only difference with a regular synchrotron emission is the lack of polarization.

\subsection{Radiation from relativistic shocks}

Relativistic flows emanate from several kinds of compact objects. They ends with strong relativistic shocks with the ambient medium. There are at least two kinds of relativistic shocks depending of the magnetization of the ambient medium characterized by the parameter:

$$
\sigma \equiv \frac{B_{\perp}^{2} / 4 \pi}{\rho c^{2}}
$$

It can be seen as the ratio of the magnetic flux that crosses the shock front over the kinetic energy flux, because the transverse magnetic field is amplified by the shock Lorentz factor $\Gamma_{s}$ at the front.

There exists a critical value of the magnetization parameter (Lemoine \& Pelletier 2010), $\sigma_{\text {crit }}$, such that for $\sigma<\sigma_{c r i t}$, intense magnetic small scale magnetic fluctuations are generated in the shock precursor and relativistic particle energization through the Fermi process. These accelerated electrons radiate through a regime that will be made precise further on. For $\sigma>\sigma_{c r i t}$, the relativistic shock is formed through the development of the Synchrotron Maser Instability. A ring of particles is produced around magnetic field lines that are responsible for an inverted population. This maser effect generates an intense coherent electromagnetic wave. The electron motions in that wave produce a strongly nonlinear IC radiation, that does not significantly differ from a synchrotron 
radiation from the viewpoint of the spectrum, however has certainly important consequence in terms of coherence effects in the radiation field.

Let us look at the low magnetization regime. Intense micro-turbulence is generated in the short precursor through beam plasma interactions. There are magnetic waves produced by Weibel instability if the magnetization is very weak, and whistler modes triggered by resonant interaction with the beam of reflected particles. There are also electronic quasi electrostatic waves generated by resonant interactions as well, with frequencies close to $\omega_{p e}$. In the precursor the electrons that are not yet fully energized by the shock undergo strong oscillations and should radiate like a synchrotron radiation. However most of the radiation is emitted by the relativistic electrons in the downstream flow, because they fill a much larger volume. These electrons oscillate in a weak regime in the intense micro-turbulence because they are highly relativistic. Indeed this can be seen as follows. In the nonlinearity parameter $a$, the thermal Lorentz factor is already very large in a proton electron plasma: $\gamma \sim \Gamma_{s} m_{p} / m_{e}$, since the temperatures are $T_{e} \sim T_{p} \sim \Gamma_{s} m_{p} c^{2}$, and electrons and protons have the same relativistic mass $\Gamma_{s} m_{p} c^{2}$. The coherence length of the micro-turbulent waves is of order of the inertial length of electrons and protons $\delta_{*} \equiv c / \omega_{p *}$ with a plasma frequency $\omega_{p *}=\left(4 \pi n e^{2} / \Gamma_{s} m_{p}\right)^{1 / 2}$. Thus

$$
a^{2}=\frac{\delta_{*}^{2}}{\bar{r}_{L *}^{2}}=\frac{\bar{B}^{2} / 4 \pi}{\rho_{d} \Gamma_{s} c^{2}}=\frac{\bar{B}^{2} / 4 \pi}{\rho_{u} \Gamma_{s}^{2} c^{2}} .
$$

A fraction $\xi_{\text {e.m. }}$ of the incoming energy is converted into electromagnetic energy:

$$
\bar{B}^{2} / 4 \pi=\xi_{e . m .} \rho_{u} \Gamma_{s}^{2} c^{2}
$$

with $\xi_{\text {e.m. }}$ of a few percent according to the numerical simulations. Thus $a^{2} \sim \xi_{\text {e.m. }} \ll 1$ but larger than $\sigma$, which means that the fluctuating field is much larger than the mean field in the downstream flow. Moreover its coherence scale is smaller than the Larmor scale, both with the fluctuating and the mean field $\left(\delta_{*} \ll \bar{r}_{L *} \ll r_{L 0}\right)$. These two sets of strong inequalities are crucial in the physics of relativistic shocks for the regime of Fermi acceleration:

$$
\begin{aligned}
\sigma & \ll a^{2}=\xi_{e . m .} \ll 1 \\
\ell_{c} \sim \delta_{*} & \ll \bar{r}_{L *} \ll r_{L 0}
\end{aligned}
$$

Despite the state of intense micro-turbulence, the electrons oscillate in a weak regime and thus radiate through a kind of IC-scattering (single scattering), that leads to an emitted spectrum similar to a synchrotron spectrum, with a characteristic frequency $\omega_{*} \sim \gamma^{2} c / \ell_{c} \sim \gamma^{2} \omega_{p *}$, a similar shape at higher frequency, but a much steeper one at lower frequencies. It turns out that all the GRBs spectra observed during both the prompt emission (with mildly relativistic shocks) and the afterglow emission (strong relativistic shock) are steeper at low frequencies than the synchrotron one $\left(\omega^{1 / 3}\right)$.

\section{9. observational challenge}

In order to explore coherence effects in the spectrum of the pulsar wind nebula such that of Crab Nebula, one needs femtoseconde correlation techniques. Indeed the spectrum reaches its 
maximum in optical band. This spectrum looks like a synchrotron spectrum and the assumption that the radiation mechanism is due a normal synchrotron emission has been widely assumed. But there is no observational evidence that this is so rather than an Inverse Compton emission through plasma waves or a coherent maser wave. Polarization measurement can bring an indication but fairly poor. Measurement of the coherence functions $g^{(1)}$ and $g^{(2)}$ would provide with a clear discrimination. In the case of a standard synchrotron emission, the function $g^{(2)}$ should be that of a Gaussian process; whereas any kind of inverse Compton radiation would provide a different result and would leas to an estimate of the nonlinearity parameter and thus the coherence length. We hope that this will become feasible in a near future.

\section{References}

[1] Jackson J.D., 1999, "Classical Electrodynamics" 3rd edition, John Wiley \& sons.

[2] Rybicki \& Ligthman, 1979, "Radiative Processes in Astrophysics", John Wiley \& sons.

[3] Melrose D., 1980, "Plasma Astrophysics" vol 1, Gordon and Breach.

[4] Kirk J. \& Reville B., 2010, arXiv:1001.0687 (astro-ph.HE).

[5] Medvedev M., 2000, ApJ, 540, 704.

[6] Rees M., 1971, Nature, 229, 312.

[7] Gun \& Ostriker J., 1971, ApJ, 165, 523.

[8] Blandford R. \& Rees M., 1972, ApJL 10, 77.

[9] Arons J., 1972, ApJ 177, 395.

[10] Lemoine M. \& Pelletier G., 2010, MNRAS, 402, 321. 